\title{
La mise en discours du savoir orthographique : que se passe-t-il dans la salle de classe?
}

\author{
Belinda Lavieu-Gwozdz ${ }^{1}$ et Thierry Pagnier $^{2}$ \\ $\mathbf{1}^{\mathbf{2}}$ : CENTRE INTERDISCIPLINAIRE DE RECHERCHE "CULTURE, ÉDUCATION, \\ FORMATION, TRAVAIL" (CIRCEFT-ESCOL (Education, Scolarisation)), Université Paris VIII \\ Vincennes-Saint Denis : EA4384, 93200 St-Denis, France \\ 2 : CENTRE INTERDISCIPLINAIRE DE RECHERCHE "CULTURE, ÉDUCATION, \\ FORMATION, TRAVAIL" (CIRCEFT-ESCOL (Education, Scolarisation)), Université Paris VIII \\ Vincennes-Saint Denis : EA4384, 93200 St-Denis, France
}

Résumé. Cette contribution s'intéresse à la manière dont le savoir est mobilisé en discours dans les interactions langagières collectives au sein de la classe, et notamment au caractère plus ou moins implicite des savoirs et connaissances grammaticales mobilisées. Cet intérêt pour les interactions collectives tient aux formes linguistiques et langagières qui construisent en discours ce collectif. Les constructions de ce collectif sont plus ou moins linguistiquement explicites et participent certainement à l'opacité ou à la visibilité des enjeux cognitifs de ces interactions. Nous nous appuyons sur un corpus de 12 à 15 séances de 5 classes qui porte sur les corrections d'exercices d'orthographe et de dictées. Dans cette première analyse exploratoire, nous chercherons à mettre en évidence les caractéristiques communes de la manière dont les savoirs sont discursivement mobilisés. Il s'agira notamment de questionner l'usage des termes du métalangage grammatical mais aussi la manière dont la justification constitue ou non un moment de stabilisation de savoirs. En effet, nous cherchons à voir si le savoir est explicité, ou au contraire si le savoir concerné donne lieu à des échanges traversés par des implicites discursifs inégalement partagés qui sont susceptibles d'obscurcir les finalités du dispositif, " réduisant 》 en quelques sortes l'activité cognitive de raisonnement grammatical à la simple exécution de la tâche de correction des formes erronées.

\begin{abstract}
Spelling Skills Speech: What Happens in the Classroom? This contribution focuses on how knowledge is mobilized in discourse in collective language interactions within the class, and especially the more or less implicit nature of the grammatical knowledge mobilised.

This interest in collective interactions stems from the language and language forms that build this collective into discourse. The constructions of this collective are more or less linguistically explicit and certainly contribute to the opacity or visibility of the cognitive stakes of these interactions.

We rely on a corpus of 12 to 15 sessions of 5 classes which deals with corrections of spelling and dictation exercises. In this first exploratory analysis, we will seek to highlight the common features of how knowledge is used discursively. In particular, it will be a question of the use of terms of grammatical metalanguage, but also of how the
\end{abstract}


justification is or is not a moment of stabilization of knowledge. In fact, we are trying to see whether the knowledge is explicit, or on the contrary whether the knowledge concerned gives rise to exchanges crossed by unequally shared discursive implicits which are likely to obscure the purposes of the device, "reducing" in a way the cognitive activity of grammatical reasoning to the simple execution of the task of correcting erroneous forms.

\section{Introduction}

Le travail présenté s'inscrit dans une recherche menée depuis deux ans au sein du CIRCEFT, un laboratoire qui centre ses préoccupations sur la question des inégalités d'apprentissage à l'école, et notamment sur le rôle du langage et des discours dans la construction de ces inégalités. Au centre de ces travaux, figurent le concept de coconstruction des inégalités scolaires (Bautier \& Rayou, 2009 ; Rochex \& Crinon, 2011 ; Delarue-Breton, 2012, 2014 et 2015). Les inégalités entre élèves, et donc les difficultés importantes, conduisant parfois à l'échec de certains d'entre eux, s'analysent comme étant le produit d'une rencontre entre des modes de socialisation familiale peu connivents des pratiques litteraciées scolaires, et des pratiques pédagogiques qui tiennent trop -ou trop peu- compte de ces écarts entre élèves au cours des activités d'apprentissage en classe.

Le concept de malentendu sociocognitif invite à prendre en compte le travail interprétatif de l'apprenant, qui risque de masquer les significations à construire.

C'est cette préoccupation qui nous a conduits à nous intéresser aux Literacy Studies et à la manière dont les concepts proposés nous permettent de mettre en évidence le sens que les élèves ont effectivement construit des situations enseignement-apprentissage de la grammaire/orthographe (Lavieu \& Pagnier, 2017).

La recherche dont nous rendons compte ici s'inscrit dans le cadre des travaux de recherche initiés dans le cadre du projet TAO « Twictée pour apprendre l'orthographe ». Ce projet E-fran a notamment pour objectif d'évaluer l'efficience et les conditions d'efficience du dispositif twictée (contraction de dictée et twitter) et d'autres manières d'enseigner l'orthographe au cycle 3.

Notre attention s'est tournée vers l'analyse des interactions en groupe classe. A une époque où l'horizontalisation des échanges pose question (Bautier, 2009), nous avons choisi de nous intéresser au discours adressé et construit en groupe classe. Ce choix est motivé par l'idée que les interactions entre pairs, sur lesquelles investissent de nombreux dispositifs pédagogiques contemporains, sont nécessairement articulées à ces moments de mises en commun des réponses et des raisonnements.

Ces interactions en groupe classe nous semblent reposer sur ce que l'on pourrait désigner comme une double illusion. D'une part, l'interaction entre quelques élèves et l'enseignant est un discours également adressé aux autres élèves de la classe. Il s'agit dès lors de se demander dans quelle mesure celui-ci est effectivement saisissable par (tous) les élèves. D'autre part, l'enseignant questionne non (simplement) pour obtenir des réponses mais pour montrer/donner à voir le questionnement que les élèves doivent s'approprier. Il s'agit dès lors de se demander comment cette finalité « seconde », souvent masquée par la place centrale des réponses dans les interactions, est donnée à voir.

Notre intérêt pour décrire les spécificités du dialogue scolaire a été suscité par le désir de mieux comprendre ce qui fait difficulté notamment pour certains élèves dont le mode de socialisation familiale est moins en adéquation avec la socialisation scolaire que d'autres élèves. Un certain nombre de travaux ont montré en effet que les difficultés récurrentes de

\footnotetext{
* https://www.twictee.org/twictee/
} 
certains élèves sont directement liées à ce qui se joue dans les échanges langagiers scolaires (voir notamment Bautier, 2008, Bautier \& Rochex, 2004, Delarue-Breton, 2017).

\section{Aspects théoriques}

Nadeau et Fisher (2011) s'intéressent aux liens entre les performances dans la réussite des accords à l'écrit et les connaissances grammaticales explicites des élèves et montrent que sont efficaces les pratiques qui attirent l'attention sur des structures, amènent à construire une conceptualisation de la langue écrite et de ses différents niveaux de fonctionnement et donc qui rendent possible un recul métalinguistique et d'autoapprentissage lors de la lecture et de l'écriture

Bonnal (2016) isole des facteurs favorisant l'apprentissage de l'orthographe et notamment la qualité de l'étayage de l'enseignant, la mise en recherche active des élèves, le choix du corpus c'est-à-dire des phrases utilisées comme support de travail, la précision du métalangage de l'enseignant, et le recours à des procédures syntaxiques plutôt que sémantiques. Notons cependant que la récente recherche de Bonnal ne constate pas d'incidence sur les progrès de tous élèves ni dans toutes les classes. A la suite de Gomila (2004, 2011, 2013), le métalangage prend des formes particulières « entre langage ordinaire et termes spécifiques et/ou métaphoriques »(Gourdet et alii 2015) qui opacifie ou au contraire explicite l'activité cognitive attendue.

La place et le rôle qu'occupent aujourd'hui les moments interactionnels dans le discours pédagogique invitent à interroger les conditions de son efficience. De nombreuses recherches ont montré l'inégale efficience des interactions lorsqu'il s'agit d'apprendre. Utiliser le langage pour apprendre nécessite effectivement la construction d'un discours second sur cet objet du monde particulier qu'est la langue. Dans un contexte d'horizontalisation des échanges (entre pairs), qu'en est-il du discours de l'enseignant adressé au groupe classe ? Selon Bernstein (2007) et Bautier (2002) tous les échanges ne se valent pas, n'ont pas de fonction cognitive d'apprentissage et tous les élèves n'ont pas les mêmes « habitus langagiers » ou modes d'usage du langage (Bernstein 1975, Lahire 2000, Pagnier 2011).

L'hypothèse qui est la nôtre ici considère qu'une telle posture seconde ne peut se construire que si les interactions langagières inscrivent les élèves dans des formes de réflexivité qui les conduisent explicitement à s'interroger sur les fonctionnements de la langue, qui vont bien au-delà de l'appropriation de règles qu'il suffirait d'appliquer.

Conformément au cadre théorique bernsteinien qui est le nôtre, nous avons voulu par le biais des notions de discours régulateur vs instructeur, identifier le caractère visible ou invisible des objectifs cognitifs des séances étudiées. La spécificité de l'institution scolaire réside dans sa fonction d'instruction : le discours instructeur y est enchâssé dans un discours régulateur qui rend le premier possible. Pour Bernstein (2007) le discours pédagogique présente la particularité d'être constitué de deux types de discours imbriqués, le discours instructeur et le discours régulateur. Le discours instructeur est celui qui contient les contenus à enseigner, et le discours régulateur est un discours d'ordre social, qui relève de la transmission des valeurs et qui crée l'ordre (ibid. : 64).

\section{Exemple de discours régulateur (DR)}

Ensei : alors les CM2 vous rangez vos deux feuilles de mathématiques - ne t'inquiète pas + ça y est les petits mots vous avez tout ce qu'il faut // des questions vous avez bien trouvé // allez on se dépêche de ranger ses 
feuilles de mathématiques et on sort son cahier de français parce qu'on va faire de l'orthographe et comme c'est vendredi, c'est le jour de la dictée allez on va s'y mettre assez rapidement ce matin s'il vous plait/ allez vous vous comportez bien

\section{Exemple de discours instructeur (DI) ${ }^{\dagger}$}

Elève 1: le loup reste calme le sujet c'est le loup

Ensei : oui de qui parle ? qui bavarde encore ?

Elève 1: le prédicat c'est reste calme le verbe c'est reste et calme c'est le complément du verbe

$[\ldots]$

Ensei : $\quad[\ldots]$ alors c'est quoi ces petits mots derrière là déjà est ce qu'ils complètent le verbe ? vous êtes sûrs que ça donne une information sur le verbe ? ça donne une information sur quoi Zachary?

Nous proposons dans le cadre de cette recherche de distinguer deux modalités complémentaires selon lesquelles se déploient le discours instructeurs: l'élaboration du savoir d'une part fait d'approximations successives (Delarue, 2019) et de tâtonnements où le savoir est en train de se construire par l'apport successif de l'interaction du groupe classe (comparaison, analogie, rapprochement dans toutes ses formes) et la stabilisation du savoir d'autre part impliquant quant à elle des jugements par catégorisations strictes, qui privilégie l'exactitude et la classification en traits nécessaires et suffisants. Cette forme de discours scolaire n'est pas un espace d'élaboration mais bien de stabilisation verbale.

\section{Exemple de discours instructeur d'élaboration (DIE)}

Ensei : dans « plongent»/ «plongeant» qu'est-ce qui ne va pas pour ce verbe ? c'est quoi le problème?

Elève 1: bah ils ont confondu le verbe plonger et le participe présent

Ensei : oui d'accord, est-ce qu'on ne peut pas être plus précis ?

Elève 2: si, moi maîtresse

Ensei : $\quad$ oui + vas-y

Elève 2: ils ont confondu le verbe plonger au présent de l'indicatif et le participe présent

Ensei: $\quad$ oui + d'accord est-ce que quelqu'un veut compléter ?

Elève 3: ils ont confondu le verbe plonger au présent de l'indicatif à la troisième personne du pluriel et le participe présent

\section{Exemple de discours instructeur de stabilisation (DIS)}

Ensei: $\quad$ comment on va justifier la terminaison de rêver ER ?

Elève : bah parce que avant le verbe rêver il y a la préposition « de » et après + le verbe + il se met toujours à l'infinitif

Ensei : $\quad$ voilà on écoute bien quand on a la présence d'une préposition (à, de, par, pour, sans, sur, sous...) et qu'on a un verbe derrière cette préposition, on le met toujours à l'infinitif en fait le problème se pose uniquement pour les verbes du premier groupe pour lesquels à l'oral on n'entend pas la différence entre infinitif et participe pour les autres + vous n'avez pas à vous poser la question par exemple il l'aide à partir rapidement et il l'aide à manger rapidement pour partir pas de problème car pour manger que la question se pose est-ce que je l'écris E ou ER et bien comme il y a la

\footnotetext{
${ }^{\dagger}$ Cet extrait relève plus précisément de ce que nous proposons d'appeler le « discours instructeur d'élaboration » (DIE).
} 
préposition à devant c'est l'infinitif on va noter ça ça c'est important c'est ce qu'il faut retenir

\section{Question de recherche et hypothèse}

L'oral polygéré qui constitue le cœur du dispositif des séances d'orthographe que nous analysons ici repose sur une co-construction des savoirs et requiert un apprentissage explicite. Evoquant les dispositifs reposant sur les pratiques langagières « horizontales », E. Bautier souligne que « le type d'échanges langagiers, inhérent à ces pratiques, qui ne fait encore que trop rarement l'objet d'un enseignement ou d'un apprentissage explicite, repose donc sur une familiarité acquise (ou non acquise, là est le problème) par les élèves, avec cette pratique socio-langagière qui consiste à utiliser le langage, les productions des pairs en particulier, pour apprendre » (Bautier, 1997 : 15). Ainsi, construire du savoir par le langage et transformer du « collectivement produit » en de «l'individuellement approprié » sont des compétences qui ne peuvent être supposées acquises ni mêmes perceptibles. Cet usage particulier de la langue pour parler de la langue est loin d'être évident et ordinaire. Pour le dire autrement, comprendre que pour écrire « maison» il faut commencer par un « $\mathrm{m}$ » et non par une porte ne va pas de soi et demande une gymnastique cognitive particulière qui modifie le rapport à la langue. La langue devient objet dont on parle et qui plus est qu'on étudie.

Cette contribution s'intéresse à la manière dont le savoir orthographique et grammatical est mobilisé en discours lors des interactions langagières collectives au sein de la classe, et notamment au caractère plus ou moins implicite des savoirs et connaissances mobilisées (Nadeau \& Fisher, 2011).

Nous cherchons à mettre en évidence les caractéristiques communes de la manière dont les savoirs sont discursivement mobilisés. Il s'agira notamment de questionner l'usage des termes du métalangage grammatical mais aussi la manière dont la justification constitue ou non un moment de stabilisation de savoirs. En effet, nous cherchons à voir si le savoir est explicité, ou au contraire si le savoir concerné donne lieu à des échanges traversés par des implicites discursifs inégalement partagés qui sont susceptibles d'obscurcir les finalités du dispositif, « réduisant » en quelques sortes l'activité cognitive de raisonnement grammatical à la simple exécution de la tâche de correction des formes erronées.

La littéracie, telle qu'elle est entendue ici est donc non seulement l'acquisition des formes écrites mais aussi un rapport à la langue, à son écriture, le produit des transformations sociales et cognitives que l'usage de l'écrit a produit dans la société et qui ont conduit à étendre le rapport symbolique et médiatisé à la langue aux objets du monde et à l'expérience elle-même, y compris dans les situations d'échanges oraux. Nous nous inscrivons donc dans la suite des New litteracy Studies, renouvelant le champ ouvert par les travaux de Goody (1979).

\section{Recueil de données et méthodologie}

Pour cette première analyse exploratoire, nous avons construit, à partir de données recueillies dans le cadre du projet TAO, un corpus qui porte sur les corrections d'exercices d'orthographe et de dictées (ou twictées).

Au-delà d'un inventaire de ressources linguistiques, il s'agit d'apprécier l'articulation de ces modes d'usages dans les interactions qui constituent le discours en classe. Nous privilégions une perspective avant tout descriptive en observant ce qui structure les 
interactions pendant les séances d'étude de la langue : quelle est la portée, la visée que les élèves et enseignants co-construisent ? Quelles sont les formes interactionnelles et discursives dans lesquels le savoir est mis en discours (questionnement, cours dialogué). Quels sont les objets de discours qui sont mobilisés ? A quel moment et comment (re)construit-on collectivement le savoir ? A quel moment et comment (c'est-à-dire sous quelles formes linguistiques) stabilise-t-on le savoir ? En quoi l'apprentissage de l'orthographe peut-il tirer parti de nouvelles formes scolaires fondées sur les échanges/les interactions en classe ? Quel espace de participation (Mondada, 2002, p. 19) se construit dans les interactions?

La présence de métatermes ne traduisant pas nécessairement une activité métalangagière ou du moins pas nécessairement celle attendue (Lavieu \& Pagnier 2017), nous nous posons donc la question des activités métalangagières et de la métalangue dans laquelle elles se réalisent et donc la portée de celles-ci dans les activités d'orthographe et de grammaire lors d'interaction collective.

Le recueil de données a été effectué par tous les membres de l'équipe. Les séances de classes ont été filmées à l'aide d'une caméra positionnée sur un plan large de sorte que les interactions soient visibles et audibles et transcrites (normes du GARS aménagées). Nous avons choisi d'observer 2 classes : une twictante c'est-à-dire une classe qui participe au dispositif twictée (des dictées via twitter) et une non twictante (c'est-à-dire une classe qui ne participe pas au dispositif twictée et qui pratique la dictée classique) lors d'une séquence de 5 séances sur l'orthographe puis trois autres classes dont une REP (Réseau d'Education prioritaire) et deux non REP (classes 16, 11 et 47). Il s'agit d'observations « écologiques ", c'est-à-dire avec l'intervention la moins sensible possible sur les manières habituelles de faire et de dire, en intervenant le moins possible dans le dispositif et l'environnement.

A ce stade, il ne s'agit pas de chercher à déplacer les pratiques mais à recueillir, à décrire les données nécessaires pour comprendre ce qui se joue dans les pratiques effectives lors de séances d'orthographe. Ce point de départ, non prescriptif et ancré dans la réalité du terrain et des questionnements professionnels nous semble constituer une dimension importante pour permettre à terme de déplacer des pratiques, des manières de dire des enseignants et des élèves.

L'objet de notre recherche est de comprendre la manière dont les élèves se saisissent de manière différenciée des situations didactiques qui leurs sont proposées dans le cadre de l'orthographe.

L'analyse de notre corpus s'organise en deux niveaux :

Le premier niveau concerne la structuration des interactions, nous avons caractérisé les interactions verbales qui structurent les séances de correction orthographique. Il s'agit de voir ce qui dans ces séances d'orthographe constitue des litteracy event autrement dit des événements de littéracie (comme la lecture des histories du soir) et d'observer des régularités au sein de ces événements pour repérer des pratiques de l'oral dans la salle de classe lors de corrections orthographiques.

Le second niveau porte davantage sur les dynamiques discursives du questionnement. Nous cherchons plus précisément ici à identifier sur quoi porte et se réalise le questionnement et comment s'articulent les différents objets de questionnement.

Notre objectif dans les exemples qui suivent n'est pas de décrire une classe particulière, une manière de faire spécifique d'un type d'enseignant mais d'illustrer des phénomènes récurrents qui se cumulent dans la pratique de l'enseignant et qui, par ces effets de cumul, peuvent gêner les apprentissages orthographiques des élèves les moins familiers socialement des usages littéraciés. C'est donc la récurrence des éléments qui peut faire obstacle aux apprentissages orthographiques des élèves.

\section{Analyses des interactions}




\subsection{Une structuration homogène des interactions}

Un premier résultat tient à la manière dont les séances sont structurées. De manière extrêmement récurrente, le texte ou la phrase à corriger structure la dynamique interactionnelle qui en suit le fil, la linéarité. Cette linéarité est parfois encadrée par des moments de rappel ou de bilan (essentiellement sur les procédures) et un discours instructeur qui précise la part pragmatique, factuelle de la tâche à réaliser. Le questionnement se déploie donc item par item en suivant la segmentation proposée par l'enseignant. Le schéma ci-dessous illustre la structuration récurrente des séances de corrections orthographiques pour quelques classes. On remarque donc que massivement, les enseignants corrigent exhaustivement toute la dictée en parcourant le texte mots après mots. Aucune classe ne propose une entrée thématique, par exemple, s'occuper dans tout le texte de la relation sujet-verbe. Le discours instructeur (bleu et orange dans notre schéma) s'entremêle de discours régulateur (en vert). On observe également au sein du discours instructeur du discours instructeur d'élaboration (en bleu dans notre schéma) très majoritaire où par tâtonnement et approximations successives les élèves parviennent à plusieurs à justifier/argumenter du choix orthographiques et du discours instructeur de stabilisation très minoritaire (en orange dans notre schéma) et non régulier. Ce discours instructeur de stabilisation se présentant comme le discours formel, la ressaisie surplombante de l'interaction qui vise une difficulté/un obstacle orthographique particulier qui a fait l'objet d'échanges.

Schéma 1. Structuration des interactions.

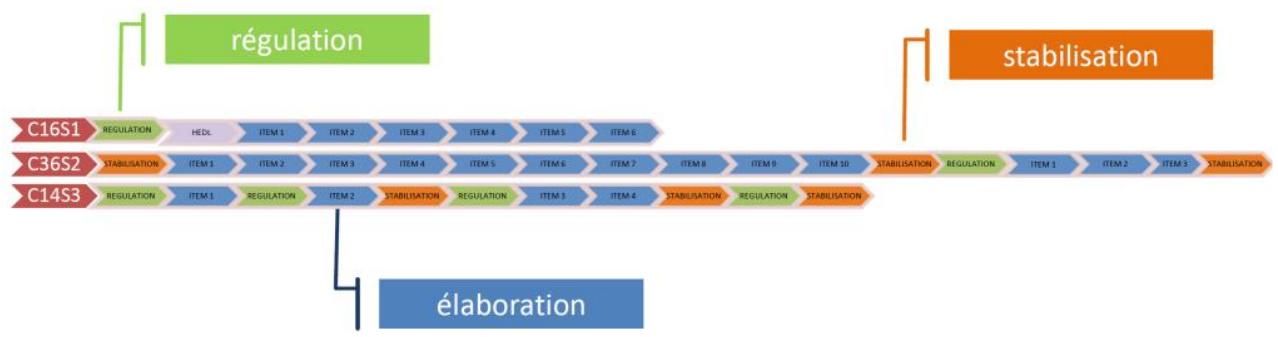

Dans notre corpus, nous identifions quatre manières de stabiliser le savoir en groupe classe :

\subsubsection{La stabilisation du savoir se fait à partir du raisonnement conduit}

M : $\quad$ nous avions affaire à un verbe, un verbe qui fonctionne avec son sujet + c'était la Grande Guerre + la Grande Guerre + on peut remplacer par « elle » sans + vous m'avez dit que « finir » était un verbe au présent et dans les souvenirs alors c'est Agnès qui nous a ressorti la règle : au présent, les verbes se finissent par I $-\mathrm{T}$.

Dans cet extrait, l'enseignante retrace les étapes chronologiques du raisonnement par lesquels les élèves sont passés pour argumenter et justifier le choix orthographiques (présence de test linguistique « remplacer », présence de récitation de règles). 


\subsubsection{La stabilisation se fait à partir des procédures}

M: ok quand je peux remplacer par un autre verbe à l'infinitif, le verbe s'écrit à ?

Charlotte : l'infinitif

On remarque ici la construction du discours de stabilisation à deux voix (maître et élève), avec la présence d'une phrase suspensive comme si l'élève devait compléter le texte à trous de l'enseignant.

4.1.3 La stabilisation se fait par métaphore, analogie, par comparaison, par rapprochement, par " air de famille » avec une catégorie grammaticale

M: $\quad$ l'adjectif il fonctionne un peu comme un caméléon + le caméléon il se pose sur une surface rouge il devient rouge il se pose sur une surface verte il devient vert l'adjectif + il va marcher exactement pareil

On remarque ici l'utilisation de la locution « un peu comme » dans son usage ordinaire de la langue. D'ailleurs l'enseignante compare la catégorie grammaticale à un caméléon mais n'évoque pas le discours scientifique c'est-à-dire les propriétés de fonctionnement de l'adjectif en termes de variation en genre et en nombre. Le paradoxe que nous dégageons et que nous exprimons en termes bernsteiniens se situe dans la construction au quotidien de la classe d'un discours " horizontal » (Bernstein 2007) et de situations " particularistes » concrètes, à traiter « matériellement » dans un oral de l'oralité ordinaire, quand l'objectif réel est celui de la construction de signification universalistes, générales qui ne peuvent se construire que dans l'élaboration que permet l'usage littéracié de la langue, y compris dans des échanges oraux. Le développement des pratiques langagières et cognitives littéraciées relève davantage de l'intériorisation que de l'explicitation. On peut ici constater que c'est en quelque sorte un savoir par approximation que le discours stabilise.

\subsubsection{La stabilisation sur les catégories grammaticales se fait à partir de son comportement syntaxique}

M : $\quad$ quand on a des des mots comme ça ce sont alors pas toujours mais enfin c'est vrai que ça ce sont des pronoms + un pronom c'est un mot qui remplace un nom + chacun il remplace un nom chacun c'est chacune des personnes chaque euh être chaque personne + c'est cela c'est c'est c'est l'éruption ça remplace l'éruption enfin le volcan sa lave meurtrière c'est tout ça que ça remplace le cela + à ce moment-là quand il s'passe ça + c'est un c'est un sauve-qui-peut général + d'accord + c'est euh donc euh pensez bien à ça hein le pronom remplace un nom

On peut ici relevé des caractéristiques linguistiques de cette phase de stabilisation, notamment l'usage du présent de vérité générale, la présence du " il » impersonnel, de pronoms comme « nous », " on », « je » au sens générique c'est-à-dire le " je » est utilisé au sens de "communauté des usagers de la langue française », des déterminant générique " un nom...le verbe... ", présence d'adverbes qui vise la généralisation " souvent ", " toujours ", " pas toujours mais très souvent », « jamais », " généralement »..., la présence du présentatif C'est/ça qui sont anaphorique introduisant des définitions : soit l'énumération des propriétés et le nom de la catégorie grammaticale associée ou l'inverse, c'est-à-dire d'abord le nom de la catégorie grammaticale à laquelle on associe les 
propriétés. On observe également pendant cette phrase de stabilisation, le recours à de nombreuses formes suspensives (indiquées dans notre transcription par des points de suspension « ....») enfin, nous remarquons dans cet extrait beaucoup d'emploi en mention, d'emploi autonymique des mots (par exemple « chaque » alors qu'on a davantage recours à des emplois déictiques (devant, ça, celui-là, etc.) dans les phases d'élaboration. Enfin, nous avons également considéré comme des " discours de stabilisation », les extraits de corpus (en faible nombre) qui mentionnait mais jamais de manière exhaustive explicitement l'opération cognitive du type, " je résume », « je reprends », « je reformule », " je répète », ainsi qu'en témoigne l'extrait suivant :

M: $\quad$ alors les CM2 je répète ce que l'on vient de dire [...]

$\mathrm{M}: \quad$ si je résume votre démonstration [...]

Thomas : on peut synthétiser en disant [...]

\subsection{Les dynamiques discursives}

Dans l'histoire de l'école, la question, c'est d'abord une condition : pour que les élèves apprennent, pour qu'ils trouvent du sens et s'engagent dans le travail scolaire » (Maulini, 2004 : 127). Le second niveau de notre analyse porte sur la dynamique discursive c'est-àdire sur le questionnement conduit pendant la séance de classe en collectif et toujours à l'occasion de correction de dictées ou exercices orthographiques. On a cherché à savoir sur quoi porte le questionnement et comment s'articulent ou s'imbriquent les différents objets de questionnement? Qu'est-ce que l'interaction construit comme objet de discours?

S'intéresser aux rapports complexes entre langage et apprentissage consiste alors à regarder comment ou en quoi le langage, entendu ici comme usages de la langue en contexte scolaire, est partie prenante de l'apprentissage orthographique. En somme, nous nous intéressons à la manière dont les moments scolaires c'est-à-dire lorsqu'on fait des exercices ou des leçons d'orthographe contribuent ou participent à construire ces représentations de ce que signifie " faire de l'orthographe ou « faire de la grammaire ». Et de manière plus générale, des représentations de ce qu'est la langue et de son fonctionnement.

L'analyse est conduite au moyen d'une grille distinguant trois types de questionnements :

a. les questions en quoi visant l'identification (par exemple, « quelle est la bonne orthographe ? », " quelle est la classe grammaticale de ce mot ? », « quel est le sens du mot $? \gg)$

b. questions en comment visant les procédures d'analyse grammaticale (par exemple, « comment je sais que ça s'écrit comme ça ?», « quel test je peux faire pour le savoir ?»)

c. questions en pourquoi visant le fonctionnement de la langue (par exemple, justification par une règle de fonctionnement de la langue).

\subsubsection{Des moments discursifs qui visent l'identification : le /QUOI/}

Dans notre corpus, nous avons donc isolé des moments discursifs qui portent sur l'identification autrement dit sur l'étiquetage des catégories grammaticales pour en déduire quelque chose sur le fonctionnement orthographique. Ce sont ces moments de discours que nous avons enregistrés dans notre catégorie /QUOI/. Nous ne parlons volontairement pas de « question en quoi » parce que nous voyons dans les extraits ci-dessous que pour cette 
catégorie "identification », nous ne nous sommes justement pas arrêtés (uniquement) aux introducteurs linguistiques. Les questions sont souvent formulées de la manière suivante par l'enseignant : «Qu'est-ce que je mets ? " «C'est quoi ? » "C'est X [morphème de pluriel pour les noms, par exemple] » ou « $\mathrm{Y}$ [morphème de pluriel pour les verbes] ». "Ça s'écrit comment ? », "C'est un [+ le nom de la catégorie grammaticale] ». La formulation de la question vise une étiquette soit du morphème, soit du graphème, soit de la nature grammaticale, elle vise la réponse orthographique, la bonne forme mais n'en vise pas l'explicitation. Ainsi dans l'extrait $n^{\circ} 1$, Juliette épèle les mots les uns à la suite des autres (quand, volcan) :

\section{Extrait $n^{\circ} 1$}

Juliette : quand le volcan

E : $\quad$ alors

Juliette : quand c'est Q.U.A.N.D

E : oui

Juliette : et le /

E : ouais non ça c'est bon j'te l'épargne

Juliette : volcan V.O.L.C.A.N

E : d'accord + on continue + vas-y euh Loan

\section{Extrait $\mathrm{n}^{\circ} 2$}

Laura: c'est vous riez

M : $\quad$ ah vous riez vous riez Lila Lilia

Lila : c'est aller au passé simple

M : $\quad$ alors aller au passé simple

Edouard : ils allèrent A deux L E accent grave R E N T

$\mathrm{M}$ : $\quad$ il va comment Dimitri euh le présent avec je Jorel

Jorel : c'est le verbe donner je donne D O deux N E

Il s'agit ici de corriger des formes orthographes mais on remarque qu'il s'agisse de corriger des items lexicaux ou verbaux, la manière de faire est la même. Ces deux extraits de classe (il s'agit de deux classes différentes), montrent que les enseignants recourent à l'épellation de la (bonne) réponse sans faire justifier ou argumenter ou découper en morphème les réponses des élèves. Ce qui prévaut dans cet échange c'est la (bonne) forme, ce qu'il fallait mettre, quelle était la bonne réponse. L'activité de raisonnement orthographique est ainsi opacifiée par l'exécution de la tâche.

Le découpage du corpus selon ces catégories montre une majorité de questions visant l'identification. On pourrait interpréter ce résultat de la manière suivante : l'orthographe est considérée comme un ensemble de savoirs ponctuels à rappeler ; ce qui est demandé aux élèves est d'étiqueter plutôt que de conceptualiser (métatermes étiquettes vs. métatermes concepts), de répondre aux questions de l'enseignant plutôt que de se questionner et raisonner sur le fonctionnement de la langue.

Les échanges qui constituent souvent l'essentiel d'une séance sont produits dans une langue quotidienne, dans un mode conversationnel de la part des enseignants comme des élèves. Ils ne représentent en effet de la part de ce dernier ni explication précise, ni définition, ni argumentation qui situerait le propos dans un apprentissage classificatoire et un usage minimal de métatermes sans que ne se déploie réellement un métalangage d'analyse. 
L'avancement du cours dialogué est fondé sur un jeu constant et très rapide de questionréponse. Nous reprenons l'expression «cours dialogué » car elle fait partie des catégories descriptives des modes pédagogiques, mais au niveau scolaire étudié, elle est abusive dans la mesure où justement, il n'y a pas toujours de " cours ", au sens de nouveau savoir à acquérir, où la visée cognitive du cours, son objet même et son maintien dans la durée ne sont pas toujours maintenus du fait des échanges eux-mêmes et de leurs caractéristiques.

Il semblerait donc que pour les élèves, ces moments d'orthographe sont tantôt perçus comme des moments d'évaluation de l'écart avec une norme qui s'impose tantôt comme une injonction à la justification orthographique mais qui se réduit à un étiquetage grammatical qui devient pour les élèves les moins connivents la finalité de l'activité plutôt qu'un moyen de penser. Cela est peut-être lié à la discipline ou la manière de se représenter le savoir orthographique mais en mathématiques la justification passe par une démonstration très normalisée qui fait l'objet d'une attention toute particulière dont on peut regretter l'absence en étude de la langue. Ce ne sont pas ici les procédures d'analyse telle que la substitution, l'effacement, le déplacement qui sont mobilisées mais la vérification dans une série de listes mémorisées.

Ils n'entrent pas dans l'activité littéraciée attendue. Les catégories ne font pas sens ou plutôt il s'agit d'étiquettes et non de catégories.

\subsubsection{Des moments discursifs qui visent la procédure : /COMMENT/}

Là encore, comme pour la catégorie précédente, la forme linguistique ne présage pas de ce qui est attendu et la forme en comment n'interroge donc pas toujours sur le comment puisque l'enseignant autorise des interventions d'élèves de différents ordres ou niveaux, ainsi que l'illustre ce premier extrait :

Extrait $n^{\circ} 1$

M : $\quad$ avec le sujet ! c'est parti Mathis ( ?) nous trouve le sujet comment on trouve le sujet dans une phrase?

Mathis : $\quad$ on dit « qui est-ce qui »?

M : $\quad$ mmmm

Mathis : $\quad$ qui est-ce qui finit? la Grande Guerre.

M : $\quad$ qui est-ce qui va finir?

Sacha: $\quad$ la Grande Guerre

M: $\quad$ se rassemblent alors tiens qui est-ce qui me rappelle pourquoi ça s'écrit «se » cette fois-ci ? allez normalement, il y a toutes les mains levées Clélia?

Alexandre : parce que derrière il y a un verbe

$\mathrm{M}$ : $\quad$ très bien Fais-moi un twoutil

Alexandre : « se » s'écrit $\mathrm{S}-\mathrm{E}$ car derrière il y a un verbe

Si l'on s'arrête sur ce premier extrait pour illustrer notre catégorie en /COMMENT/, on remarque (en gras dans l'extrait) que l'enseignante formule aussi bien une question en /comment/ (Comment on le trouve le sujet dans une phrase ?) qu'une question en /pourquoi/ (Pourquoi ça s'écrit SE). On pourrait alors supposer que les attentes ne sont pas les mêmes. Or, si l'on regarde les réponses des deux élèves Mathis et Alexandre, toutes deux conviennent à l'enseignante (ou du moins aucun écart n'est signalé). Ainsi, bien que la forme de la question soit différente linguistiquement la réponse des élèves vise dans les deux cas la procédure. Dans le premier cas, Mathis énonce, récite une procédure (la question sémantique en qu'est-ce que ?) pour identifier le sujet et dans le second cas, 
Alexandre mobilise une procédure de repérage, de localisation c'est parce que y a un verbe derrière «se » qu'on l'écrit SE mais le terme de « verbe pronominal » n'apparait pas.

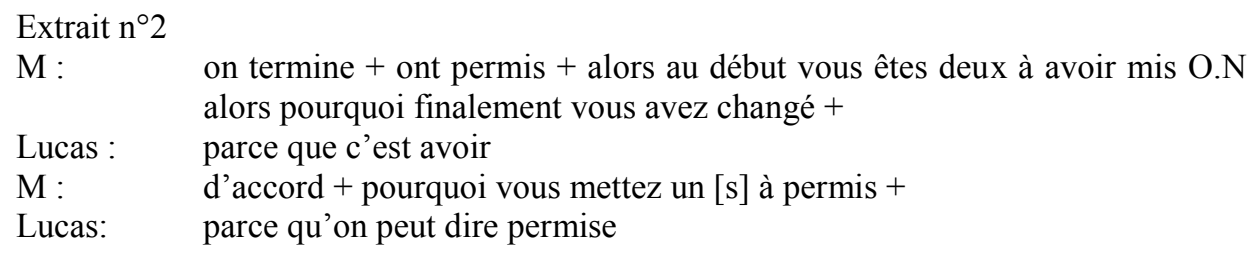

La première question formulée en pourquoi ne relève pas de notre catégorie visant le fonctionnement de la langue /POURQUOI/ dans la mesure où elle vise davantage l'identification, c'est-à-dire la (bonne) réponse de ce qu'il faut ou non mettre. De plus ici on remarque l'absence d'explicitation. A la question pourquoi il fallait mettre ONT et non ON, l'enseignante se contente de la réponse en " parce que c'est avoir » sans demander aux élèves d'expliciter ce que signifie leur réponse « parce que c'est avoir ». Il est fort probable que pour l'élève peu connivent cette réponse ne fait pas sens et ne prend pas sens dans le système qu'est la langue. Par ailleurs, la réponse de l'élève pour justifier de la présence du $\mathrm{S}$ à permis est totalement opaque et n'est pas redépliée pour que la procédure énoncée le soit explicitement. Prise telle que, la réponse peut conduire certains élèves peu connivents, à conclure que permis s'écrit toujours P.E.R.M.I.S et que la conjugaison du verbe permettre à la troisième personne du singulier sous cette forme Elle permit, par exemple, n'existerait pas. Dans les deux cas de figure on reste dans l'implicite et aucun des exemples n'est reconfiguré dans le système orthographique du français par exemple pour distinguer le fonctionnement du verbe permettre et celui du participe ou le fonctionnement du pronom réfléchi et celui du déterminant démonstratif.

\subsubsection{Des moments discursifs qui visent le fonctionnement de la langue : /POURQUOI/}

Dans cette catégorie de discours, nous avons regroupé des extraits d'interactions qui visent des questionnements de type " pourquoi » c'est-à-dire que l'enseignante demande de justifier, d'argumenter les réponses en recourant au fonctionnement du système qu'est la langue, d'en convoquer son mode de fonctionnement. Ainsi, nous avons sélectionné ici des questions du type « Pourquoi ce mot prend un « s » et pas « -ent»»? L'enseignante invite les élèves à mobiliser leur connaissance sur la langue et notamment, par exemple sur l'opposition de catégorie grammaticale telle que noms vs verbes ou encore sur l'opposition nom propre et nom commun comme l'illustre l'extrait suivant :

Extrait $n^{\circ} 1$ :

M: $\quad$ alors + la grande Guerre nous + quand on a réfléchi au texte, on l'a écrit avec des majuscules + alors pourquoi on l'a écrit avec des majuscules + Max?

Léa : $\quad$ parce que c'est la première guerre mondiale

M : $\quad$ c'est le nom qu'on a donné à la première guerre mondiale alors ça vous ne pouviez pas le savoir parce qu'en histoire vous n'êtes pas encore là mais on l'a appelée souvent on dit tiens c'est la Grande Guerre alors la Grande Guerre malheureusement c'est parce que c'est la guerre où il $\mathrm{y}$ a eu le plus de morts alors on l'appelle la Grande Guerre et si on l'a appelée comme ça, ça devient du coup un nom ++

Léa: $\quad$ propre

M : $\quad$ ça devient un nom propre. 
Au fil de l'échange, on observe des objets de discours (plus ou moins proches du domaine " orthographe ») qui s'articulent -plus ou moins explicitement- en suite, en imbrication, dans des niveaux différents d'organisation du discours.

Extrait $n^{\circ} 2$ :

M : $\quad$ qu'est-ce que vous aviez à saigner ce matin ça va ça va + alors vous gardez vos cahiers de brouillon et dans vos cahiers de brouillon en principe vous m'avez fait des phrases pour ce matin // alors hum hum on est prêt le cahier de brouillon les lunettes Emeline c'est mieux Zachary tes lunettes aussi on est assis correctement alors quel était qu'est-ce qu'on a fait hier matin quelle était la leçon de hier matin allez on a travaillé sur quoi + Rodrigue

Rodrigue : l'attribut du sujet

M : on a travaillé sur l'attribut du sujet qu'est-ce que c'est qu'un attribut du sujet qu'est-ce que c'est Thaïs

Thaïs : déjà sa nature c'est soit un adjectif soit un groupe nominal

M : $\quad$ c'est soit un adjectif soit un groupe nominal oui

Paul : $\quad$ et il s'accorde en genre et en nombre avec le sujet

M : $\quad$ il s'accorde en genre et en nombre avec le sujet

Luvine: il est placé après le verbe

M : $\quad$ il est placé après le verbe Louane

Louane : et surtout cela complète le sujet

M : d'accord ça complète le sujet ça donne des informations sur comment est le sujet oui

Lucas : $\quad$ il est toujours placé derrière un verbe d'état

Laura : elle l'a déjà /

M : $\quad$ un verbe d'état qu'est ce que c'est qu'un verbe d'état

Thaïs : $\quad$ non elle a dit /

$\mathrm{M}$ : $\quad$ elle a dit après le verbe

Rodrigue : oui c'est un verbe d'état c'est un verbe qu'on a du mal à mimer c'est un peu compliqué donc je suis mimer être c'est /

M : $\quad$ oui d'accord oui

Léa : $\quad$ le verbe d'état on peut le remplacer par être

M : $\quad$ le verbe d'état on peut le remplacer par être ça c'est vrai c'est très bien estce que vous les connaissez un petit peu les verbes d'état les principaux

Isaure : oui

Mathis : oui

M : $\quad$ ils étaient dans la leçon ça va mieux va vite à ta place Zachary

Luvine: a l'air

$\mathrm{M}$ : $\quad$ allèrent

Zachary: non mais a l'air

M : $\quad$ ah avoir l'air avoir l'air excuse-moi excuse-moi c'était pas du tout oui avoir l'air oui mettez le moi à l'infinitif s'il-vous-plait parce que là je suis comme vous je suis fatiguée aussi moi + Rodrigue

Rodrigue: il y a avoir l'air sembler

M : oui

Léa : être

M : oui

Thaïs : demeurer

M : oui

Lucas : devenir

M : oui 
Paul : $\quad$ il y a aussi paraître

Du fait que les élèves emploient les métatermes « féminin », « nom », « pluriel », « verbe », etc. on tire (souvent à tort) l'idée qu'ils savent ce que c'est et comment fonctionne la notion de pluriel, de féminin, etc. quel que soit le mot ou le constituant syntaxique. Or, les interactions verbales analysées ici et les entretiens métagraphiques -qui feront l'objet d'une prochaine publication- font apparaitre que les méta-termes font très peu l'objet de questionnement dans la salle de classe ou bien lorsque c'est le cas ce sont principalement des définitions sémantique «le verbe, c'est l'action » qui y sont attachées. Quand on suit de près les interactions en classe au moment de la correction de dictées, twictées ou exercices orthographiques, on s'aperçoit de très nombreuses approximations, de glissements sémantiques, de confusions qui passent souvent inaperçues -qui en tout cas ne sont pas explicitées par les enseignants- mais qui témoignent d'une construction notionnelle largement encore inachevé pour beaucoup d'élèves. On retrouve là l'analyse de Vygotski sur la différence entre les mots utilisés par les enfants et les adultes : s'ils désignent les mêmes objets et permettent de communiquer, leur signification et les opérations cognitives mises en jeu ne coïncident pas toujours.

Ce travail de reprises, de redites, ces verbalisations qui examinent les objets de discours sous toutes les coutures, ces bribes de raisonnement pas à pas, les retours en arrière passagers, ces graphies transitoires c'est bien ce qui permet de construire comme appartenant aux mêmes classes et de rassembler sous une même étiquette des éléments très divers. Tant que ce travail d'élaboration n'est pas achevé, les élèves n'ont pas recours à la métalangue en tant qu'elle construit des catégories mais les élèves plaquent des connaissances, convoquent des listes mentales d'appartenance ou de non-appartenance à la classe et utilisent majoritairement les mots en mention. C'est ici une autre source de l'inefficacité de cet enseignement qui fait de la règle et de la métalangue le tout de la connaissance orthographique, alors qu'elles sont surtout le point d'arrivée (et non le point de départ). Si pour comprendre et assimiler l'orthographe, les élèves ont besoin de passer par des interactions verbales, il faut alors pouvoir répondre, organiser, structurer, prévoir cette nécessité dans le discours de la classe même.

\section{Bilan de l'analyse des interactions}

Dans les interactions en classe, au moment de corrections de dictées ou d'exercices orthographiques, on constate des questionnements qui visent le /comment/, c'est-à-dire qui visent la procédure auxquels la réponse par /quoi/ est autorisée, des questionnements qui visent le /quoi/, c'est-à-dire qui visent l'identification, l'étiquetage auxquels la réponse en /comment/ est autorisée ainsi que des questionnements qui visent le /pourquoi/, c'est-à-dire qui visent à l'élaboration des connaissances sur le système du fonctionnement de la langue auxquels la réponse par /quoi/ est autorisée. On note donc que le type de réponses ne correspond pas nécessaire à une forme précise de questions, ce qui participe certainement à opacifier les objets du questionnement conduit « collectivement ».

Quantitativement, les questionnements visent très massivement l'identification, l'étiquette, la (bonne) réponse. Peu de questionnement en /comment/ qui mobilisent des procédures ou alors principalement des procédures sémantique (qui est-ce qui ? pour repérer le sujet a question quoi ou qui ? pour repérer le COD du verbe, ou encore à qui ? à quoi pour repérer le COI du verbe) très peu de manipulations syntaxiques (suppression, déplacement, extraction en c'est...que, etc.) sont mobilisées pour construire des connaissances sur le fonctionnement des constituants de la phrase. On constate également peu de questionnement en /pourquoi/ c'est-à-dire qu'on n'observe pas de reconfiguration des réponses orthographiques dans le système linguistique, le système de la langue. On ne montre pas aux élèves le comportement syntaxiques des catégories grammaticales les unes par rapport aux autres. Il n'y a donc pas d'articulation routinière identifiable à laquelle les 
élèves peuvent s'attacher pour répondre tantôt sur l'identification, tantôt sur la procédure, tantôt sur la manière de reconfigurer leur réponse orthographique dans le système plus générale qu'est le fonctionnement de la langue.

A titre d'exemple, sur une séance de 45 minutes d'orthographe, 35 minutes concernent la correction de la dictée. En 35 minutes, 13 items (en moyenne) sont passés en revue. Ce temps d'interaction compte 23 questionnements répartis très inégalement (cf. schéma 2), c'est-à-dire des questionnements sur le QUOI autrement dit comme nous l'avons vu plus haut sur l'identification, l'étiquetage et de manière très minoritaire sur le POURQUOI c'est-à-dire sur le fonctionnement de la langue vu comme un système et le COMMENT qui permet aux élèves d'énoncer, de verbaliser, d'expliciter des procédures.

Schéma 2. Structuration détaillée des interactions.

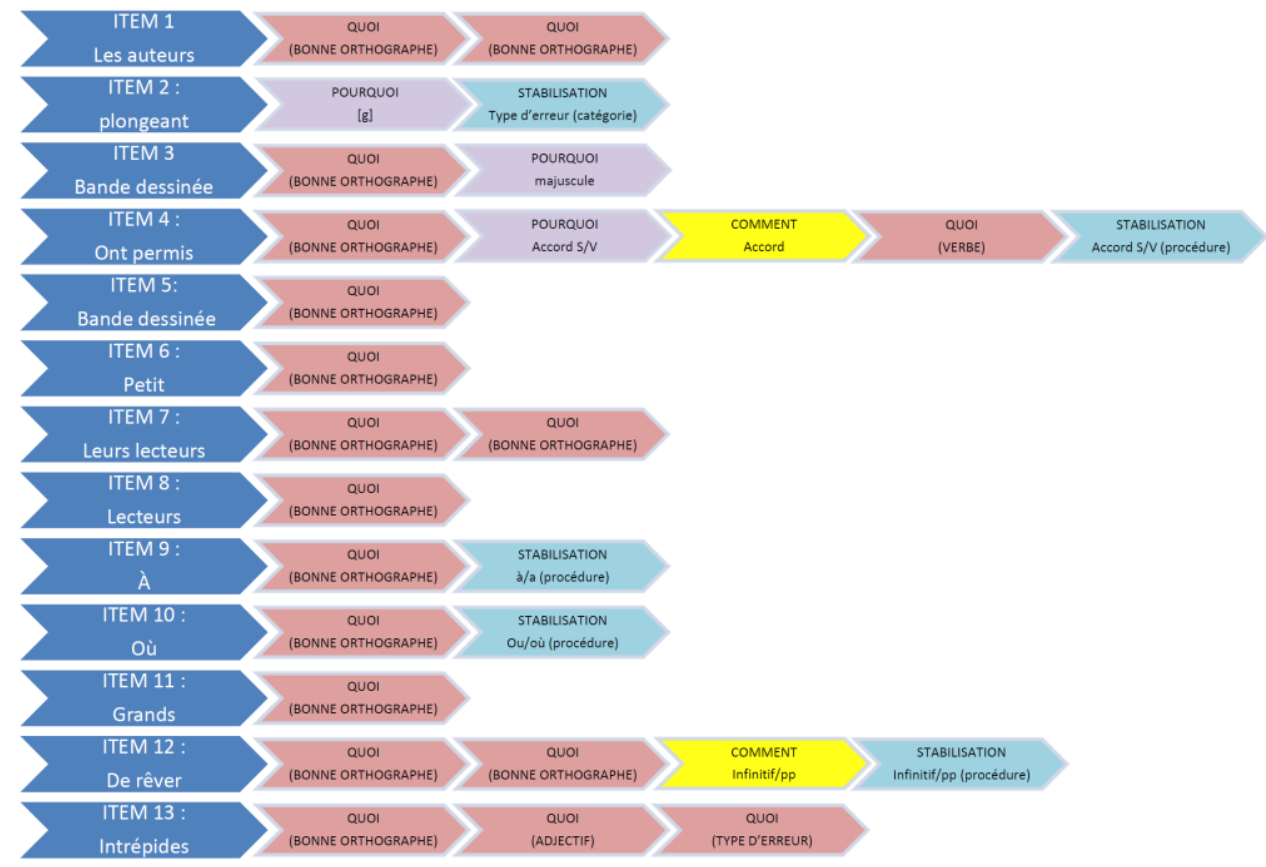

Nous faisons l'hypothèse que ce sont ces habitudes cognitives et scolaires, durablement installées, qui gênent voire empêchent les apprentissages orthographiques (et grammaticaux) dans le long terme.

A travers ces extraits de classe, on peut opposer deux manières de se représenter la langue : une première où on représente la langue comme un système avec ses régularités (analogie, distinction,...) et une seconde, majoritaire, où la langue ne fait pas système mais est alors perçue comme un ensemble de pièges auxquels on peut faire face à la condition de connaitre les « astuces », les règles et « contre-exemples ». Sans compter que cette seconde manière de se représenter la langue est souvent doublée d'un rapport normatif à la langue et à ses usages (il faut dire/ne pas dire) qui est particulièrement prégnant en France (Lodge, 1997). Ainsi les règles de fonctionnement qui font de la langue un système sont présentées soit comme réservées à des initiés en mesure de rétablir l'implicite discursif soit comme en dehors du champ des apprentissages envisageables. Le point de vue défendu ici est que toutes les formes discursives ne favorisent pas les apprentissages scolaires, notamment disciplinaires, et que certains vont même jusqu'à les invisibiliser. 
En bref, la forme de la question ne présage pas de la forme de la réponse et donc on a bien des questionnements sur le /COMMENT/ auquel on répond par QUOI... des questionnements sur QUOI auquel on répond par COMMENT et aussi des questionnements sur le POURQUOI auquel on répond par QUOI. Les élèves ne peuvent donc pas s'emparer, se fier à la forme de la question pour donner une réponse plutôt qu'une autre. L'appropriation du questionnement lui-même en est donc compliquée. A l'issu de l'analyse de notre corpus, nous ne sommes pas parvenus à identifier un format, un moule du questionnement. On peut penser que la formalisation en twoutils permet de pallier ces impasses dans la mesure où les twoutils sont des éléments qui permettent de formaliser à l'écrit le cadre dans lequel se fait la justification orthographique. L'analyse des twoutils sera l'objet d'une future publication que nous rapprochement de la présente analyse des interactions de classes.

\section{Conclusion}

Au-delà des compétences du lire-écrire, c'est en effet un rapport réflexif sur la langue qui est attendu lors des séances d'orthographe.

En l'occurrence tous les apprenants sont en mesure de placer la langue en position d'objet de discours. Toutefois l'activité orthographique et/ou grammaticale (visée) implique de faire de la langue un objet mais un objet d'un type particulier. Un objet d'analyse, c'està-dire un objet sur lequel va se développer un discours qui vise la construction de signification. En somme, lors de ces séances d'orthographe, il s'agit de développer un discours « théorisant » sur la langue au sens de Bautier (2002) et Delarue-Breton (2012), c'est-à-dire une analyse cognitivement "outillée » de procédures de vérification et de catégories d'analyse. L'exemple le plus parlant est celui de l'orthographe lexicale qui est renvoyée à un apprentissage " par cœur ». Par exemple, l'accent circonflexe de fête ou d'hôpital est souvent présenté aux élèves comme étant à retenir " photographiquement» sans que jamais la systématie de la chute du «s $»$ que l'on retrouve dans les dérivés ne soit convoquées festival, hospitalier soit qu'elle n'est pas connue, soit qu'elle n'est pas mobilisable ou qu'elle semble inaccessible en dehors d'un raisonnement étymologique hors de portée. Dans tous les cas, le système lexical n'est pas présenté comme tel c'est-à-dire structuré par des relations formelles et sémantiques entre les unités.

Nous défendons l'idée que les pratiques mobilisées relèvent de la littératie lorsqu' "ils font de la grammaire ou de l'orthographe » mais que les compétences littératiées mobilisées relèvent d'une littératie ordinaire et non de la littératie scolaire (Lavieu \& Pagnier 2017).

Cette recherche montre également que pour une part importante des élèves l'activité grammaticale et/ou orthographique demeure une activité tendue par une finalité que l'on qualifierait volontiers d'autotélique (Belin, 2002 ; Delarue-Breton, 2012) tant elle s'épuise dans la réalisation même de l'activité : faire de la grammaire, c'est étiqueter.

L'appariement se réalise sur la base de la vérification dans une liste mentale d'éléments que les élèves donnent aux éléments stéréotypiques de cette catégorie. Ainsi, la catégorie « nom » regroupe les éléments désignant des choses, la catégorie « sujet » regroupe des éléments qui désignent celui qui fait l'action, la catégorie « verbe » indique l'action et ainsi de suite.

Cette procédure d'étiquetage relève bien d'un usage littéracié de la langue. Pour autant elle s'écarte de l'activité d'analyse que vise l'enseignement orthographique, notamment aujourd'hui.

En accédant à l'écrit et en apprenant l'orthographe, les élèves n'apprennent pas seulement un nouveau savoir mais entrent dans de nouvelles sphères d'échanges et s'engagent dans un autre rapport au monde, au rapport où le langage devient objet et 
instrument de connaissance dans une communauté culturelle et discursive fondée sur la culture écrite (Vygotski 1937/1997, Lahire 1993 ; Olson 1994, Bernié, 1994).

On enseigne essentiellement l'orthographe comme un savoir, pourtant c'est en tant que savoir-faire que le niveau orthographique des élèves est véritablement évalué. La mixité de l'orthographe française avec des phénomènes tels que la plurifonctionnalité des graphèmes, la morphographie en partie silencieuse et combinée à une grande part d'homophonie/hétérophonie, suffisent à expliquer l'impossibilité d'une maitrise rapide de l'orthographe française. Effectivement, il y a une rupture entre connaître des règles d'orthographe et mettre en œuvre des procédures orthographiques.

L'analyse de notre corpus montre que massivement, l'interaction verbale ne vise pas la construction de connaissances orthographiques mais l'étiquetage et la bonne réponse. Lorsqu'il y a argumentation sur la justification orthographique, celle-ci est souvent lacunaire, très implicite et aucun moule sur la manière de justifier orthographiquement ne guide la démonstration. Or, le travail d'explicitation que requièrent les interactions verbales amène l'élève à revenir sur son action, à déployer pour l'autre ses procédures et les objets sur lesquels portent celles-ci. De ce fait, l'élève a une certaine prise sur ses propres opérations mentales et peut exercer un certain contrôle. Un travail de transformation s'effectue en direct. En explicitant ses savoirs et ses doutes, l'élève construit discursivement des objets linguistiques en l'occurrence les constituants de la notion d'accord en genre, amené à rapprocher des éléments d'ordre lexical, graphique, morphologique syntaxique il manipule des propriétés et progresse du concept encore flou de féminin vers celui de genre. Pourtant l'intervention de l'adulte n'apporte que trop peu souvent les savoirs nécessaires. Le dépliage, le raisonnement se fait implicitement mené par certains élèves qui vont mettre en relation des séries et des catégories qu'ils sont en train de construire. Ce parcours notionnel très implicite dans le discours pédagogique constitue pourtant l'acte d'apprendre que tous les élèves sont appelés à s'approprier.

\section{Références}

Bautier, E. (1997). Les pratiques socio-langagières dans la classe de français ? Quels enjeux ? Quelles démarches? Repères 15.

Bautier, É. (2001). Pratiques langagières et scolarisation. Revue française de pédagogie, 137, 117-161.

Bautier, É. (2002). Du rapport au langage : question d'apprentissages différenciés ou de didactique? Pratiques 113-114, 41-54.

Bautier, É. (2005). Formes et activités scolaires, secondarisation, reconfiguration, différenciation sociale. Dans N. Ramognino et P. Vergès (dir.), Le français hier et aujourd'hui : Politiques de la langue et apprentissages scolaires. Hommage à Viviane Isambert-Jamati (pp. 49-68). Aix-en-Provence : Publications de l'Université de Provence.

Bautier, É. (2008). Ce que l'école fait aux individus. Colloque de Sociologie de l'éducation dans le cadre de la journée scientifique de l'Université de Nantes : "Pratiques de travail et pratiques langagières scolaires : constructions au quotidien des inégalités sociales d'apprentissage ».

Bautier, É. (2009). «Quand le discours pédagogique entrave la construction des usages littéraciés du langage », Pratiques : Écrits et savoirs, n ${ }^{\circ}$ 143-144, p.11-26.

Bautier, É. \& Rochex, J.-Y. (2004). Activité conjointe ne signifie pas signification partagée », Raisons éducatives, 8, (p. 199-220).

Bautier, E. et Rayou, P. (2009). Les inégalités d'apprentissage : programmes, pratiques et malentendus scolaires. Paris, France : Presses universitaires de France 
Bernié, J.-P. (2002). L'approche des pratiques langagières scolaires à travers la notion de « communauté discursive » : un apport à la didactique comparée ? Revue française de pédagogie, 141, 77-88.

Bernstein, B. (1975) Langage et classes sociales, codes socio-linguistiques et contrôle social. Paris, Les Editions de Minuit.

Bernstein, B. (2007). Pédagogie, contrôle symbolique et identité : théorie, recherche, critique / traduit par Ginette Ramognino-Le Déroff \& Philippe Vitale. Sainte-Foy [Québec] : Presses de l'université Laval.

Bonnal, K. (2016). L'orthographe telle qu'elle s'enseigne : pratiques d'enseignement de l'accord sujet-verbe observées à la fin de l'école primaire, thèse de doctorat (sous la direction de C. Garcia-Debanc).

Cogis D. et Ros Dupont, M. (2003). Les verbalisations métagraphiques : un outil didactique en orthographe?, Dossiers des sciences de l'éducation 9, 89-98.

Delarue-Breton C. (2012). Dispositifs et logiques dispositives : perception des enjeux et inégalités scolaires. In M.-L. Elalouf, A. Robert, A. Belhadjin \& M.-F. Bishop (Dir.), Les didactiques en question : état des lieux et perspectives pour la recherche et la formation. Bruxelles : De Boeck, 120-130.

Delarue-Breton C. (2014). Dialogue scolaire, dialogisme et évènements d'énonciation : malentendus et inentendus au sein de la classe. Études de Linguistique Appliquée, 173, 8797.

Delarue-Breton C. (2015). D'un document à l'autre : circulation des savoirs et déambulations différenciées des élèves. Didactiques en pratique, 1, 18-24. [Disponible en ligne] ]

Delarue-Breton, C. (2017). Activité dialogique à l'école : observations sur l'élaboration des significations au sein du dialogue scolaire. Recherches en didactiques des langues et des cultures, 14-2. [En ligne] : http://rdlc.revues.org/1875

Delarue-Breton, C. (2019). Le dialogue scolaire, un genre discursif frontalier, dans Raisons éducatives 23.

Gomila, C ; (2004). «Pratiques d'enseignement de la langue au CP : Quelle place pour les savoirs grammaticaux dans l'enseignement de la lecture? ». In Vargas C. (dir.) Langue et étude de la langue. Approches linguistiques et didactiques. Aix-en-Provence : Publications de l'université de Provence, p. 291-300.

Gomila, C. (2011). Parler des mots, apprendre à lire. La circulation du métalangage dans les activités de lecture. Berne : Peter Lang.

Gomila, C. (2013). « Le petit mot de la phrase : entre catégorisation pratique et classification grammaticale ». In Bertrand O., Schaffner I. (dir.). Enseigner la grammaire. Palaiseau : les Éd. de l'École polytechnique, p. 145-158.

Goody, J. (1979), La raison graphique, Paris, Minuit.

Gourdet, P. , Gomila, C., Bourhis V., Elalouf M.-L. , Péret, C. et Avezard-Roger, C. (2015). De la grammaire au CP pour lire et écrire ? Description et analyse de pratiques dans le cadre de la recherche Lire-écrire au CP, Repères 52.

Lahire B. (2000). Pour une sociologie psychologique de la famille, Presses Universitaire de France.

Lahire B. (1993). Culture écrite et inégalités scolaires. Sociologie de l'échec scolaire à l'école primaire, Lyon : PUL.

Lavieu, B. et Pagnier, T. (2017). Quelles pratiques littéraciées sont mobilisées par les futurs professeurs des écoles lorsqu'ils « font de la grammaire » ?, Lidil 56 [disponible en ligne https://journals.openedition.org/lidil/4806\#text]

Maulini, O. (2004). Un enjeu invisible de l'innovation pédagogique : l'institution du questionnement. Dans : Jean-Paul Bronckart éd., Transformer l'école (pp. 127-146). Louvain-la-Neuve, Belgique: De Boeck Supérieur. doi:10.3917/dbu.bronc.2004.01.0127 
Mondada, L. (2002). Cognition et parole-en-interaction, Veredas, Revista de Estudos Linguisticos, 6/1, 9-27.

Nadeau, M. et Fisher, C. (2011). Les connaissances implicites et explicites en grammaire : quelle importance pour l'enseignement ? Quelles conséquences ? Bellaterra Journal of Teaching \& Learning Language \& Literature, 4(4), 1-31.

Pagnier, T. (2011). "Quelle "langue de départ" avant la transmission scolaire de la "norme standard" ? La nomination des bruits, des odeurs, et des couleurs », Variétés, variations et formes du français, O. Bertrand, I. Schaffner (dir), Editions de l'école polytechnique, p.295-309.

Rochex, J.-Y et Crinon, J. (dir.) (2011). La construction des inégalités scolaires. Au cœur des pratiques et des dispositifs d'enseignement, Presses Universitaires de Rennes.

Vygotski L. (1934). Pensée \& Langage, réédition (1997) Paris : La Dispute. 